\title{
Large Deformation Numerical Simulation of the Set up Effect Induced by Sand Compaction Pile Group Installation
}

\author{
Chenyu Kang ${ }^{1, *}$, Jiangtao $\mathrm{Yi}^{1}$ \\ ${ }^{1}$ School of Civil Engineering, Chongqing University, Chongqing 400045, China.
}

\begin{abstract}
The improvement of soil undrained shear strength induced by installation of sand compaction pile (SCP), called set up effect, has observed in a number of field tests, but not been considered by the design code. Due to the lack of effective technical means, the numerical simulation of this problem mainly focused on single pile in the past studies. In this paper, a multi-stage Eulerian-Lagrangian (MSEL) technique is used to simulate the installation and consolidation process of double piles. Firstly, a centrifuge model test is used to benchmark this technique and reached a good agreement. Through the analysis of a single pile results, the strength growth curve conforming to the growth law is fitted. Finally, based on the results of single and double piles and considering the sheltering and accumulating effect, a method to predict the soil strength change after SCP group installation is proposed.
\end{abstract}

\section{Introduction}

Sand compaction pile (SCP) is a kind of foundation treatment technology with fast construction speed, wide application range and obvious reinforcement result. The traditional SCP method originated from Europe in the 1830s. Due to the lack of construction equipment, it did not develop rapidly at home and abroad until the 1950s. With the invention of the machine, it has been widely used in recent years. Especially in Asian, there are some famous projects, such as Marine bay in Singapore, Busan New Port in Korea, the Yangshan Deepwater Port and the Hong Kong-Zhuhai-Macau Bridge in China. Because the clay is not removed before SCP construction, its installation will lead to soil deformation and generate a large amount of excess-pore pressure. Subsequently, as it dissipates the effective stress and undrained shear strength will increase, named set-up [1]. This phenomenon has been observed in numerous field and centrifuge model tests [1-4]. However, the research on this effect is still insufficient, resulting in the inability to consider existing design specifications [5]. Numerical simulation of it faces great challenges. The main difficulties are: 1. Large soil deformation can easily cause mesh distortion, and the calculation results cannot converge. 2. The complicated process is difficult to simulate. In previous research, scholars usually use a cylindrical expansion model to simplify the SCP installation. Besides that, there are other methods, like removing and reactivating elements [6], using "dummy material" [7], the cluster volumetric expansion feature [2] or movement of two rigid bodies [1]. Based on an axisymmetric model, only a single pile can be considered in the above method. However, as a composite foundation, multiple piles need to be further considered. In this paper, multi-stage EulerianLagrangian (MSEL) technique is applied to analyse the soil strength improvement due to two SCPs installation. Centrifuge model data of SCP installation were first used to benchmark numerical analyses. Based on the results of single pile, the curve of post-installation soil undrained shear strength was fitted. Finally, compared with the double pile, a method to predict the strength after the installation of pile group was proposed.

\section{Numerical model}

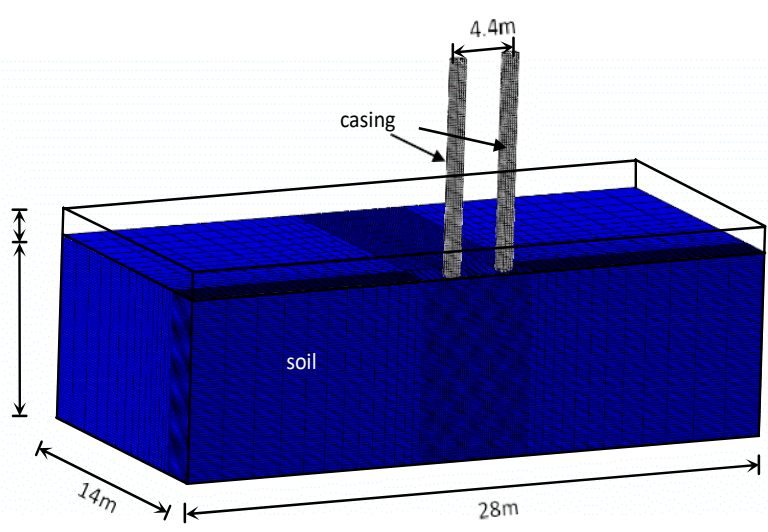

Fig. 1. Geometry and Eulerian mesh of the 3D model

\subsection{Multi-stage Eulerian-Lagrangian technique}

The investigations in this study were conducted with multi-stage Eulerian-Lagrangian technique (namely 
MSEL), which shows good capabilities in solving complex geotechnical problems. This technique is based on the coupled Eulerian-Lagrangian (CEL) technique in ABAQUS [8], and small deformation analysis is added to analyse consolidation process. The analyses were carried on using commercially software ABAQUS V6.14.

\subsection{Model parameter}

As shown in Figure 1, half of the model is used in this study due to symmetry. Soil domain was discretized as 8-node linear Eulerian brick elements (EC3D8R). After the model size sensitivity study, the model with a length of $28 \mathrm{~m}$, a width of $14 \mathrm{~m}$ and a height of $10 \mathrm{~m}$ is sufficient to eliminate the boundary effect. To upgrade computational efficiency, a gradient mesh is used. The refined zone is a cubic element with a side length of 0.12 $\mathrm{m}$, and the casing and pile adopt a four-node double curved thin shell element (S4R) with a radius of $0.35 \mathrm{~m}$. The casing is set as a rigid cylindrical shell, while the SCP shell is assumed to be a linear elastic cylindrical shell. The deformation behaviour of the SCP is determined by the user subroutine VDISP. The Modified Cam-Clay model were adopted to consider Singapore marine clay. The parameters of SCP and clay [9] are summarized in Table 1.

Table 1. The value of parameters for numerical simulation

\begin{tabular}{|c|c|c|}
\hline Material & Parameters & Value \\
\hline \multirow{7}{*}{$\begin{array}{l}\text { Singapore } \\
\text { marine } \\
\text { clay }\end{array}$} & $\begin{array}{c}\text { Effective Poisson's ratio, } \\
v^{\prime}\end{array}$ & 0.3 \\
\hline & $\begin{array}{c}\text { Critical state frictional } \\
\text { constant, } \mathrm{M}\end{array}$ & 1.0 \\
\hline & $\begin{array}{c}\text { Gradient of swelling } \\
\text { line, } \kappa\end{array}$ & 0.066 \\
\hline & $\begin{array}{c}\text { Gradient of compression } \\
\text { line, } \lambda\end{array}$ & 0.27 \\
\hline & $\begin{array}{l}\text { Intercept of critical state } \\
\text { line, } \Gamma\end{array}$ & 3.63 \\
\hline & $\begin{array}{l}\text { Over consolidation } \\
\text { ratio, OCR }\end{array}$ & 1.0 \\
\hline & $\begin{array}{l}\text { Effective unit weight of } \\
\text { soil, } \gamma^{\prime}\left(\mathrm{kN} / \mathrm{m}^{3}\right)\end{array}$ & 5.6 \\
\hline \multirow{2}{*}{ SCP } & $\begin{array}{l}\text { Young 's modulus, E } \\
(\mathrm{kPa})\end{array}$ & 27000 \\
\hline & Poisson's ratio, $v$ & 0.3 \\
\hline
\end{tabular}

\subsection{Simulation step}

The installation process is divided into two steps: casing penetration and sand compaction (Figure 2 (a)). First, the casing is penetrated from the surface of the soil to the bottom through the displacement control casing. Then, as shown in Figure 2 (b), in the user subroutine VDISP, the nodes of the sand pile expand to the pile radius $\left(R_{s}\right)$ at the same radial velocity $\left(\mathrm{v}_{\mathrm{e}}\right)$.

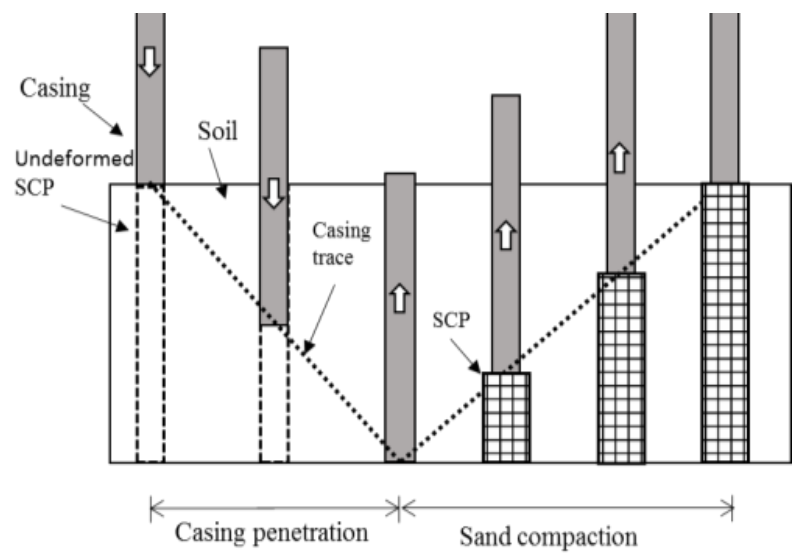

(a) single SCP construction

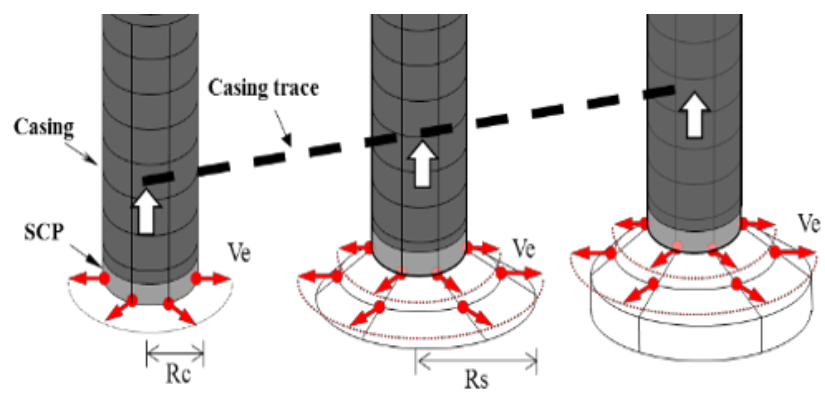

(b) detail of sand compaction step

Fig. 2. Schematic illustration of simulation method

\subsection{Contact}

Due to the high piling speed and the low consolidation coefficient of marine clay, the undrained assumption is reasonable. The "general contact" algorithm was adopted to describe two pairs of contacts: between casing and soil from beginning, and between SCP and soil after the penetration step. In order to ensure the stability of the numerical calculation, the surface is assumed to be smooth. 


\section{Validation}

In order to verify the accuracy of the MSEL calculation module, the numerical calculation results were compared with Juneja's centrifuge model [3]. As shown in Figure 3, during the casing penetration, both the pore pressure and the total stress gradually increase. There is a peak before reaching a constant value. This phenomenon has also been observed in many in situ tests of piles, also as displacement piles due to cavity expansion and stress bubble generation around the casing tip. The calculation results indicate that the MSEL calculation module has a good capability in dealing with soil large deformation questions.

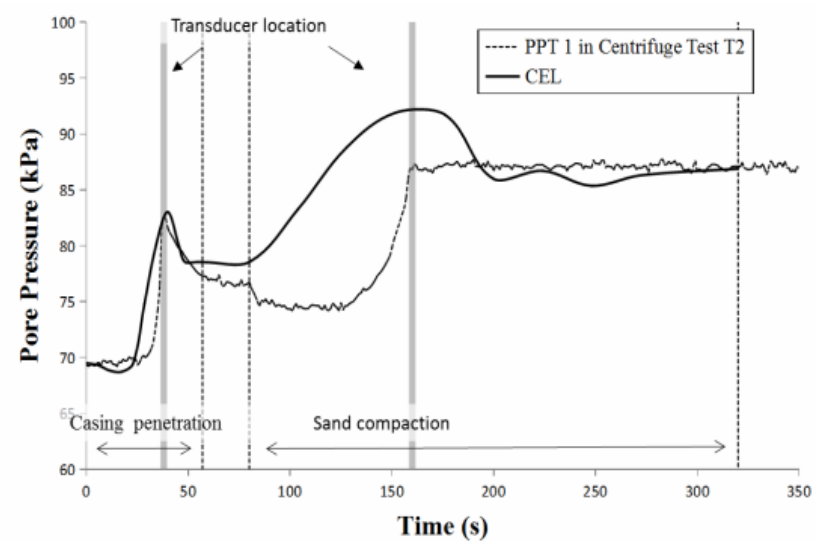

(a) PPT1 in Test2

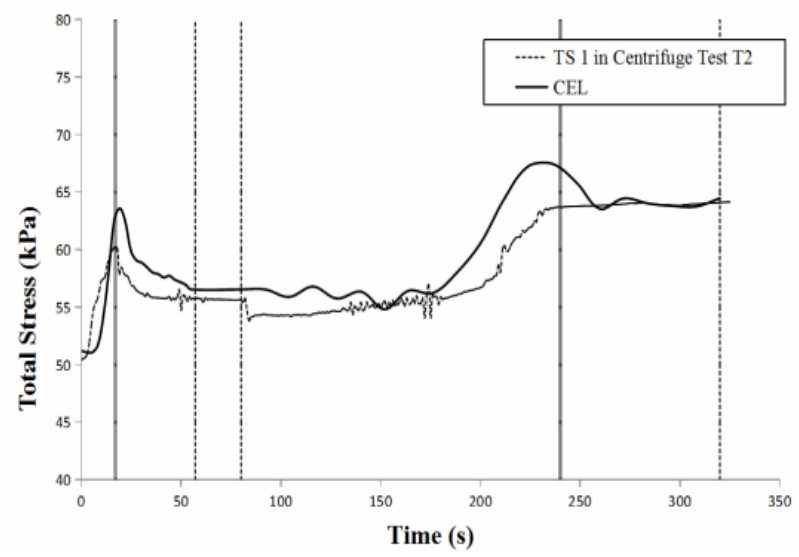

(b) TS1 in Test2

Fig. 3. Comparison of measured and computed stress during SCP installation

\section{Single pile result}

\subsection{Time effect}

The installation of SCPs can cause huge pore water pressure in the soil. Due to the existence of sand, the drainage path is shortened and the consolidation speed is accelerated. As shown in Figure 4, after the completion of the pile installation, the pore water pressure generates then dissipates after consolidation. Around the SCPs, the top and bottom was set as a drainage boundary. Moreover, the dissipating speed is uneven, and the dissipating is obvious, and it stabilizes with the development of time. After 3 years, excess pore water pressure gradually dissipates to completion.

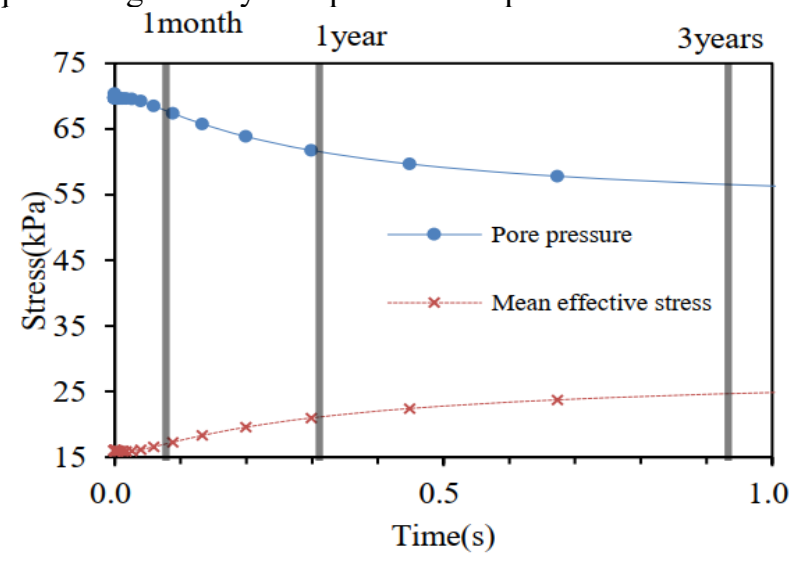

Fig. 4. Computed pore pressure and average stress at different time

\subsection{Height effect}

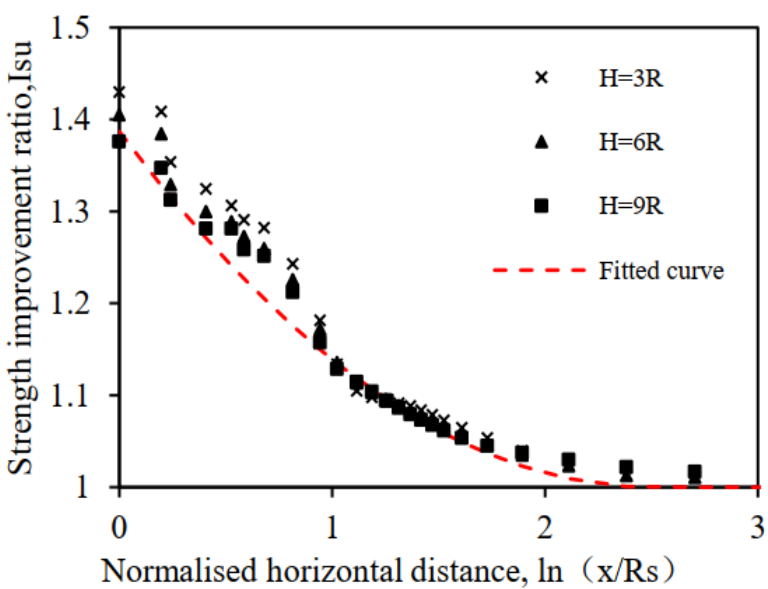

Fig. 5. Computed strength improvement ratio $I_{s u}$ for a single SCP at different depths

The dissipation of pore water pressure brings about an increase in the mean effective stress. From the relationship between the mean effective stress and soil shear strength, it indicates the strength of the soil increases. Figure 5 shows the distribution of soil strength growth with the pile centre distance after consolidation calculation. The strength increase curves at different depths basically coincide, and the influence range is 8 times the SCP radius. The distribution curve can be reflected by following curve:

$I_{s u}^{1 p}=\left\{\begin{array}{cc}0.045\left[\ln \left(\frac{x}{R_{S}}\right)-2.8\right]^{2}+1, \ln \left(\frac{x}{R_{S}}\right) \leq 2.8 \\ 1 & , \ln \left(\frac{x}{R_{S}}\right)>2.8\end{array}\right.$

in which, $I_{s u}=\frac{s_{u}}{s_{u 0}}$ represents the improvement ratio of soil undrained shear strength 


\section{Double pile result}

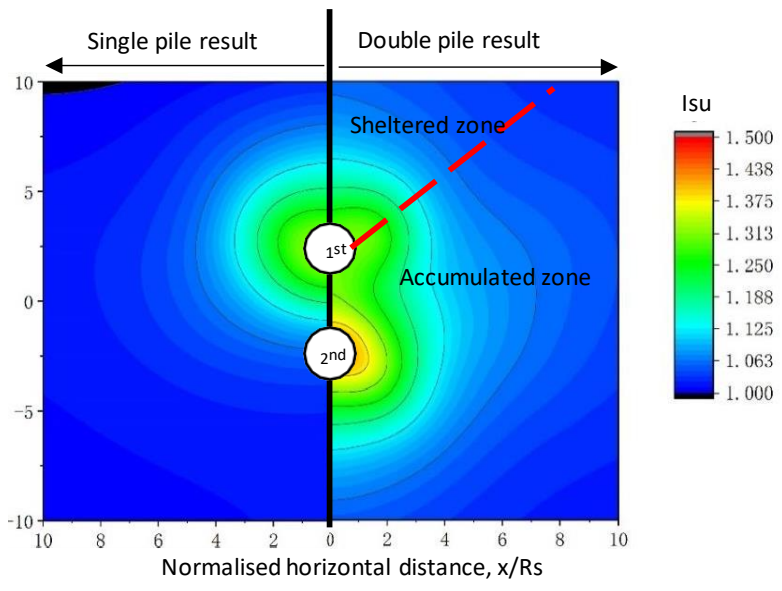

Fig. 6. Computed strength improvement ratio $I_{\text {su }}$ contour

As mentioned-above, the distance between the two piles was $\mathrm{s}=4.4 \mathrm{~m}$ (about $5.5 \mathrm{R}_{\mathrm{s}}$ ). If a square row pile method is used, the replacement area ratio will be $10 \%$, which is a low replacement rate. Figure 6 shows the distribution of strength improvement caused by single and double piles at height of $5 \mathrm{~m}$. It can be seen that due to the symmetry, the contour of single pile shows concentric circles around the pile centre.

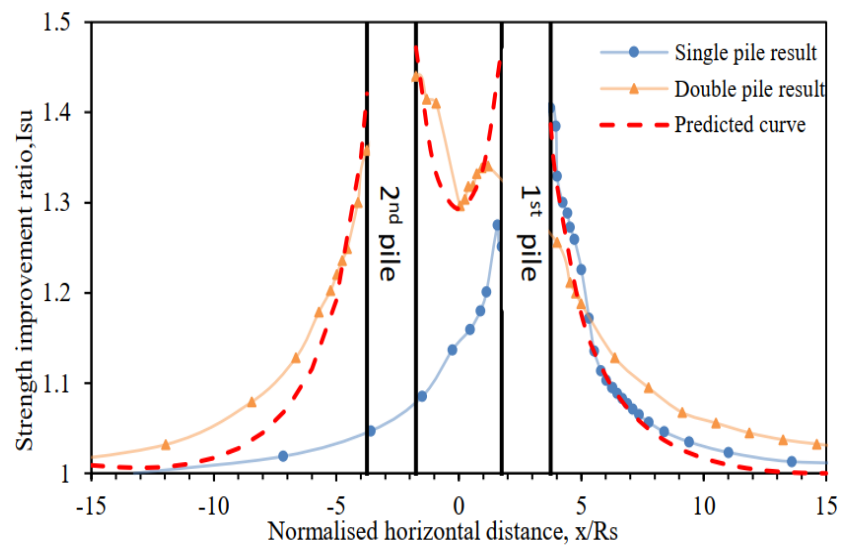

Fig. 7. Computed and predicted strength improvement ratio $I_{s u}$

After driving into the second pile, the strength of surrounding soil is change obviously. Among them, the soil strength around the second pile and the connection between the two piles are the highest (over 1.4 times initial value). On the back side of the 1 st pile, the soil strength has almost no increase due to the sheltering effect. In view of this feature, the results of the double pile can be predicted by the fitting curve of the single pile, as shown in Figure 7, which is well compounded with the calculation results of the two piles. Based on this result, for SCP group, a formula (2) is proposed to predict the post-installation undrained shear strength.

$I_{s u}^{n p}=\left\{\begin{array}{cl}I_{s u}^{(n-1) p} * I_{s u}^{n t h} & , \text { accumlated zone } \\ I_{s u}^{(n-1) p} & , \text { sheltered zone }\end{array}\right.$

\section{Conclusion}

In this paper, an advanced large deformation technique (MSEL) is used to investigate the set up effect induced by two SCPs. Validated by a centrifuge experiment, the results show that this technique can effectively simulate the complex installation and consolidation process of SCPs. From the results of a single SCP, the increase in soil strength decreases with the pile centre distance, and are uncoupled with its depth. For the growth brought about by two SCPs, the soil strength enhancement is superimposed, especially around the SCP and the connection area between the two piles. Due to the sheltering effect, the back side of the first pile is almost unaffected. This paper also puts forward a formula to predict the soil undrained shear strength after SCP group installation, which is able to consider the accumulating effect and the sheltering effect.

\section{References}

1. Yi J T, S H Goh and F H Lee, Effect of sand compaction pile installation on strength of soft clay Geotechnique 63 no 12: 1029-41 (2013)

2. Weber T M, S M Springman, M Gäb, V Racansky and H F Schweiger, Numerical modelling of stone columns in soft clay under an embankment In Proc 2nd Int Workshop on Soft Soils: Focus on Ground Improvement 305-11 Glasgow (2008)

3. Juneja A, Centrifuge model study of the effects of sand compaction pile installation on soft clay ground PhD National University of Singapore (2002)

4. Aboshi H, E Ichimoto, M Enoki and K Harada, The composer - a method to improvecharacteristics of soft clays by inclusions of large diameter sand columns In Proceedings of the International Conference on Soil Reinforcement: Reinforced Earth and Other Techniques 211- 16 Paris (1979)

5. Asaoka A, T Kodaka, M Nozu and T Hirao, Ed Pande GN and Pietruszczak S, Increase of undrained shear strength and anisotropic characteristics of clay ground improved with sand compaction piles Numerical models in geomechanics: (1995)

6. Farias M M, T Nakai, H M Shahin, D M Pedroso, P G O Passos and M Hinokio, Ground densification due to sand compaction piles Soils and Foundations 45 no 2: $167-80$ (2005)

7. Guetif Z, M Bouassida and J M Debats, Improved soft clay characteristics due to stone column installation Computers and Geotechnics 34 no 2: 104-11 (2007)

8. Qiu G, S Henke and J Grabe, Application of a coupled eulerian-lagrangian approach on geomechanical problems involving large deformations Computers and Geotechnics 38 no 1: 30- 39 (2011)

9. Xiao W H, Yielding and failure of cement treated soil PhD National University of Singapore (2009) 